\title{
Realização experimental da simulação do algoritmo de Deutsch com o interferômetro de Mach-Zehnder
}

\author{
Experimental realization of the simulation of Deutsch's algorithm using Mach-Zehnder interferometer \\ Pedro Henrique Grosman*1@, Daniel Gonzaga Braga ${ }^{1}$, Jose Augusto Huguenin ${ }^{1}$ \\ ${ }^{1}$ Instituto de Ciências Exatas, Departamento de Física Universidade Federal Fluminense, Volta Redonda, RJ, Brasil
}

Recebido em 08 de Julho, 2018. Revisado em 02 de Setembro, 2018. Aceito em 10 de Setembro, 2018.

\begin{abstract}
Neste trabalho apresentamos a realização experimental da simulação do algoritmo de Deutsch através da montagem de um interferômetro de Mach-Zehnder, conforme proposta de Cabral e colaboradores 20. O aparato montado permite a entrada simultânea dos dois qbits, conforme o circuito de Deutsch. Os resultados evidenciaram uma limitação desta interpretação. O interferômetro de Mach-Zehnder simula resultados de saída do circuito de Deutsch devido ao controle da diferença de fase entre os braços do interferômetro, independente do segundo qbit. Apesar do experimento ter sido realizado com um feixe intenso, mostramos a concordância dos resultados obtidos com o previsto para o caso de fótons únicos. Os resultados estão em sintonia com muitos trabalhos que utilizam feixes intensos para explorar princípios de informação e computação quântica, mostrando ser um cenário promissor para discussão e ensino de aspectos fundamentais da mecânica quântica.

Palavras-chave: computação quântica, interferência quântica, paralelismo quântico.
\end{abstract}

We present in this work the experimental realization of the simulation of Deutsch's algorithm using a MachZehnder interferometer setup, as previously proposed by Cabral and coauthors 20. Our setup allows the simultaneous entrance of two qbits, as in Deutsch's circuit. The results showed a limitation of this interpretation. The Mach-Zehnder interferometer simulates output results of Deutsch's circuit due to the control of phase difference between the arms of the interferometer, independently of the second qbit. Although our experiment was realized using a intense beam, we showed the concordance of our results with the expected results for unique photons. Our results agree with many works that use intense beams to explore the fundamentals of quantum information and quantum computation. It is an interesting scenario to discuss and teach fundamental aspects of quantum mechanics.

Keywords: quantum computing, quantum interference, quantum parallelism.

\section{Introdução}

Já é conhecido que o processamento de informação por meio de sistemas quânticos mostra-se mais poderoso em comparação aos sistemas clássicos [1]. Considerando as vantagens da computação quântica em relação à computação clássica, a principal motivação na busca pela construção de um computador quântico eficiente é devida a capacidade que este sistema apresenta de resolver alguns problemas que são impossíveis de serem resolvidos de maneira clássica [2]. Em outros casos, também é possível que o computador quântico solucione de forma mais eficiente um problema que já possui solução clássica 3 [4]. Um simples exemplo desta admirável propriedade é objeto deste trabalho.

Desde que a ideia da invenção de um computador quântico começou a tomar forma, três décadas atrás [2, 5], muitos estudos interessantes vêm sendo realizados nesta proveitosa linha de pesquisa que une ciência da computação, física e matemática. Uma variedade de algoritmos

*Endereço de correspondência: grosmanp@fisica.ufmg.br quânticos 6 e técnicas de implementação [1] já foram propostas. Atualmente conhecemos alguns sistemas físicos que são candidatos para futuramente compor um computador capaz de realizar operações quânticas. Dentre estes, os mais estudados são: átomos 7], moléculas [8] e luz 9 .

Os ingredientes necessários para se fazer computação quântica, como superposição de estados, interferência e emaranhamento, que serão explorados mais detalhadamente neste texto, podem ser emulados na óptica clássica. As similaridades entre a óptica paraxial e mecânica quântica não relativística $[10$ são levadas em conta para descrever a óptica em termos do formalismo de Dirac [11 e usar a álgebra de operadores para solucionar problemas de maneira elegante 12]. De fato, um feixe laser pode ser descrito tanto como um campo eletromagnético clássico ou como um estado coerente contendo um número macroscópico de fótons. Por isso, experimentos de óptica linear feitos com feixes intensos apresentam comportamentos que reproduzem muitos resultados feitos com fótons únicos. A coerência óptica é o principal 
fator que permite esta analogia que tem sido utilizada em muitos trabalhos de pesquisa. Por exemplo, na violação de desigualdades quânticas com feixes intensos como as desigualdades de Bell para sistemas bipartidos 13,14 e a desigualdade de Mermim para sistemas tripartidos [15], bem como a simulação de portas lógicas 16 .

Do ponto de vista didático, o estudo de protocolos de informação quântica tem potencial para aplicação de conceitos quânticos, oportunizando a compreensão desta importante teoria. Trabalhos com esta proposta tem sido produzidos pela comunidade 17,20 . Já o uso de ferramentas de óptica clássica foi recentemente utilizado para realizar a simulação do protocolo BB84 de distribuição de chaves quânticas [21], em um experimento simples de largo alcance didático.

Em 1985 Deutsch propôs uma sequência de operações quânticas capazes de resolver um problema matemático impossível de ser resolvido com o uso de operações ou métodos clássicos [2]: saber se uma dada função booleana, que possui somente dois valores de entrada e de saída, é constante ou balanceada, executando-a uma única vez. Sabemos que, classicamente, esse teste precisa de, no mínimo, duas execuções.A fim de apresentar este problema em nível didático, Cabral et al. [20] apresentam, do ponto de vista teórico, uma interpretação do algoritmo de Deutsch à luz do interferômetro de Mach-Zehnder.

Neste trabalho apresentamos a realização experimental da simulação do algoritmo de Deutsch. Como primeiro passo apresentaremos os fundamentos necessários para compreensão da linguagem de circuitos quânticos (Seção 2) bem como uma explicação mais detalhada sobre o algoritmo proposto por Deutsch (Seção 3). Descreveremos o interferômetro de Mach-Zehnder (IMZ) na Seção 4. Em seguida, na Seção 5, faremos um mapeamento do circuito de Deutsch no interferômetro e mostraremos que estes possuem a mesma estrutura matemática $[22$. O principal objetivo deste trabalho está em mostrar, experimentalmente, que o sistema físico em questão, possui as propriedades necessárias para a simulação do algoritmo de Deutsch e, possivelmente, outros algoritmos quânticos sem a necessidade de preparação de estados puramente quânticos da luz. Na seção 6 sumarizamos as principais conclusões deste trabalho.

\section{Fundamentos de informação quântica}

Uma vasta literatura que aborda de forma clara e detalhada as bases teóricas e experimentais da mecânica e informação quântica já é existente $[1,23,24$. Ainda assim, faremos uma breve introdução sobre estes tópicos.

\subsection{Representando a informação}

Um bit, menor unidade de informação que se pode armazenar ou transmitir, é um conceito fundamental da teoria clássica da computação. De forma análoga, a teoria quântica de informação construiu o conceito de quantum bit, ou simplesmente qbit. Assim como um bit clássico possui um estado físico, os possíveis estados para um qbit, análogos ao caso clássico, são representados pelos vetores de estados $|0\rangle$ e $|1\rangle$.

A mais importante diferença entre estes dois sistemas está no fato de que, graças às propriedades fundamentais da mecânica quântica, os qbits podem estar em um estado diferente de $|0\rangle$ e $|1\rangle$. É possível que este qbit esteja em uma combinação linear dos vetores de estados $|0\rangle$ e $|1\rangle$, também conhecida como superposição. Desta maneira, um qbit é matematicamente bem representado pela expressão:

$$
|\psi\rangle=\alpha|0\rangle+\beta|1\rangle
$$

onde os coeficientes $\alpha$ e $\beta$ são números complexos tais que $|\alpha|^{2}+|\beta|^{2}=1$, uma vez que este estado deve estar normalizado. Desta forma fica claro que, do ponto de vista matemático, o estado de um qbit nada mais é do que um vetor pertencente a um espaço vetorial complexo de duas dimensões.

\subsection{Circuitos Quânticos}

Assim como no caso clássico, circuitos quânticos também devem possuir portas lógicas responsáveis pela manipulação da informação. Uma porta lógica quântica é representada, matematicamente, por uma operação unitária realizada sobre um estado quântico que o leva para um outro estado. Como estamos falando de operadores em mecânica quântica, estas portas lógicas devem ser representadas por matrizes.

Uma porta lógica de 1 qbit, muito útil e de fundamental importância na computação quântica é a conhecida porta Hadamard,

$$
H \equiv \frac{1}{\sqrt{2}}\left[\begin{array}{rr}
1 & 1 \\
1 & -1
\end{array}\right] .
$$

Esta porta é responsável por criar superposição e leva os estados da base computacional, $|0\rangle$ e $|1\rangle$, em $(|0\rangle+|1\rangle) / \sqrt{2}$ e $(|0\rangle-|1\rangle) / \sqrt{2}$, respectivamente. A superposição de estados é muito importante na computação quântica pois é através dela que temos o chamado paralelismo quântico [1]. Nas seções seguintes veremos como a Hadamard é de extrema importância neste trabalho.

A Figura 1 mostra a representação gráfica do circuito que exemplifica o funcionamento da porta Hadamard. A linha cheia indica a evolução temporal de um dado qbit, da esquerda para direita. A caixa indicada com a letra $\mathrm{H}$ é o circuito que implementa a porta Hadamard. A linha cheia na sequência da caixa indica a evolução do qbit resultante após a atuação da porta no qbit de entrada.

Agora que uma breve apresentação dos fundamentos necessários foi feita, vamos de fato apresentar o problema central que desejamos abordar neste texto. 


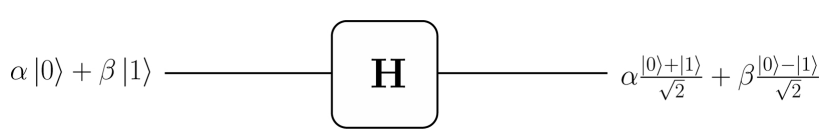

Figura 1: Porta lógica $H$.

\section{Problema de Deutsch}

Além das referências já citadas, uma boa e curta introdução ao problema de Deutsch é feita por Kinne [26]. Ainda assim, examinaremos aqui este problema de maneira mais detalhada.

Imagine uma função booleana genérica $f:\{0,1\} \rightarrow$ $\{0,1\}$, a qual desejamos examinar sua imagem e descobrir se esta é constante ou balanceada. Uma vez que esta função possui dois valores de entrada e saída, temos quatro possibilidades a serem consideradas.

A resposta para este problema é bem simples. Basta executar esta função duas vezes para entradas diferentes e comparar os valores de saída. Uma maneira de fazer esta comparação é, por exemplo, calcular a soma binária destas saídas:

$$
f(0) \oplus f(1)=\left\{\begin{array}{l}
0, \text { se } f \text { constante } \\
1, \text { se } f \text { balanceada }
\end{array}\right.
$$

A princípio esta parece ser uma solução impossível de ser otimizada já que, classicamente, precisaríamos calcular esta função duas vezes para então executar uma comparação. Porém, um computador quântico é capaz de solucionar este problema executando $f$ apenas uma vez, fazendo uso do paralelismo e da interferência quântica. Estes dois termos serão explorados com mais detalhes a seguir, quando apresentarmos uma variação do algoritmo originalmente proposto por Deutsch [2]. Apesar desta solução não possuir aplicações de grande importância, o problema de Deutsch foi pensado para exemplificar a maneira na qual podemos utilizar circuitos quânticos para construir computadores e como as propriedades da mecânica quântica podem ser interessantes para o futuro da computação. Com este mesmo propósito apresentamos este trabalho.

Deutsch, em 1989, publicou um trabalho intitulado por "Quantum Computational Networks" 27. Em seu texto, dentre outras coisas, o autor introduz a teoria das redes quânticas computacionais como uma generalização quântica dos circuitos lógicos usados nos computadores clássicos. Com a introdução da nova linguagem, que já usamos na seção anterior, seu algoritmo teve ampla repercussão pois a nova linguagem introduzida por Deutsch era similar à linguagem de circuitos lógicos/digitais convencionais.
Na Figura 2 abaixo apresentamos um circuito que implementa, de forma simplificada, o algoritmo de Deutsch. Para isto preparamos dois qbits, um no estado $|0\rangle$ e outro no estado $|1\rangle$. Assim, o registrador quântico é inicialmente representado pelo vetor de estado $\left|\Psi_{0}\right\rangle=|01\rangle$.

A operação unitária $U_{f}$ determina a computação de uma função $f$ e age sobre dois registradores da seguinte forma:

$$
U_{f}(|x\rangle|y\rangle)=|x\rangle|y \oplus f(x)\rangle .
$$

Perceba que não falamos sobre a forma explícita de $f$ e nem sobre o sistema físico que implementa a operação $U_{f}$ mas sim, apenas assumimos que existe um circuito lógico capaz de agir sobre um registrador quântico da mesma maneira que na Eq. (4).

Depois da aplicação das duas portas $H$, conforme o circuito da Figura 2, o estado do sistema será:

$$
\left|\Psi_{1}\right\rangle=\left[\frac{|0\rangle+|1\rangle}{\sqrt{2}}\right] \otimes\left[\frac{|0\rangle-|1\rangle}{\sqrt{2}}\right] .
$$

Agora, vamos analisar com cuidado as possibilidades para os valores de $f(x)$. Se

$$
\begin{aligned}
\left|\Psi_{1}\right\rangle & =\frac{1}{2}[|0\rangle+|1\rangle] \otimes[|0\rangle-|1\rangle] \\
& =\frac{1}{2}[|00\rangle-|01\rangle+|10\rangle-|11\rangle]
\end{aligned}
$$

então, de acordo com a Eq. (4), a aplicação de $U_{f}$ sobre $\left|\Psi_{1}\right\rangle$ fornece:

$$
\left|\Psi_{2}\right\rangle=\left\{\begin{array}{l} 
\pm\left[\frac{|0\rangle+|1\rangle}{\sqrt{2}}\right]\left[\frac{|0\rangle-|1\rangle}{\sqrt{2}}\right], f(0)=f(1) \\
\pm\left[\frac{|0\rangle-|1\rangle}{\sqrt{2}}\right]\left[\frac{|0\rangle-|1\rangle}{\sqrt{2}}\right], f(0) \neq f(1)
\end{array}\right.
$$

Para enxergarmos este resultado com mais facilidade precisamos levar em conta cada uma das quatro funções $f(x)$ possíveis, conforme Tabela 1, e executar as contas.

Tabela 1: Quatro funções tipo $f:\{0,1\} \rightarrow\{0,1\}$.

\begin{tabular}{c|c|c|c|c}
\hline$x$ & $f_{0}(x)$ & $f_{1}(x)$ & $f_{2}(x)$ & $f_{3}(x)$ \\
\hline 0 & 1 & 0 & 0 & 1 \\
1 & 1 & 0 & 1 & 0 \\
\hline
\end{tabular}

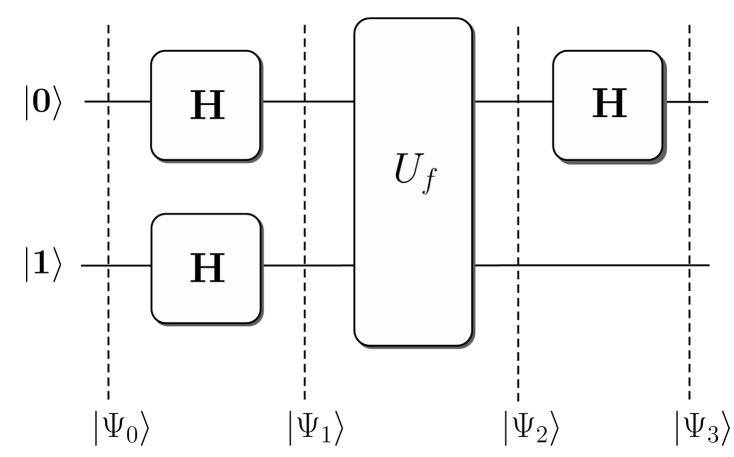

Figura 2: Circuito de Deutsch. 
Como exemplo mostraremos um caso particular onde $f(0)=0$ e $f(1)=1$. Os estados $\left|\Psi_{0}\right\rangle$ e $\left|\Psi_{1}\right\rangle$ permanecem os mesmos para qualquer que seja $f(x)$, já que a preparação do estado inicial $(|01\rangle)$ e a aplicação de $H$ são independentes da forma de $f(x)$. Com esta aplicação particular de $U_{f}$ sobre o registrador $\left|\Psi_{1}\right\rangle$ temos:

$$
\begin{aligned}
\left|\Psi_{2}\right\rangle & =U_{f}[|00\rangle-|01\rangle+|10\rangle-|11\rangle] \\
& =[|00\rangle-|01\rangle+|11\rangle-|10\rangle] \\
& =[|0\rangle-|1\rangle] \otimes[|0\rangle-|1\rangle]
\end{aligned}
$$

onde, por simplicidade, foi omitido o fator $1 / 2$ responsável pela normalização. Veja que este resultado se encaixa perfeitamente no caso em que $f(0) \neq f(1)$ como mostrado na Eq. (7).

Como segundo exemplo faremos um outro caso particular onde $f(0)=1$ e $f(1)=1$. Os passos a serem seguidos são os mesmos que anteriormente.

$$
\begin{aligned}
\left|\Psi_{2}\right\rangle & =U_{f}[|00\rangle-|01\rangle+|10\rangle-|11\rangle] \\
& =[|01\rangle-|00\rangle+|11\rangle-|10\rangle] \\
& =-[|0\rangle+|1\rangle] \otimes[|0\rangle-|1\rangle]
\end{aligned}
$$

Este resultado também coincide com uma das possibilidades para $f(0)=f(1)$ mostradas na Eq. (7). As outras duas possibilidades podem ser feitas facilmente de maneira completamente análoga.

Aqui é importante notar que uma única aplicação de $U_{f}$ age sobre todos os vetores de estado que compõem o registrador quântico $\left|\Psi_{1}\right\rangle$. De uma só vez, esta operação é capaz de calcular todos os valores possíveis para $f(x)$. Esta característica é chamada de paralelismo quântico, termo empregado por Deutsch para distinguir este conceito do paralelismo clássico, onde vários processadores se unem para executar, ao mesmo tempo, uma tarefa.

Voltando para a Eq. (7), o próximo passo é realizar uma aplicação de $H$ ao primeiro qbit deste registrador:

$$
\left|\Psi_{3}\right\rangle=\left\{\begin{array}{l} 
\pm|0\rangle \otimes\left[\frac{|0\rangle-|1\rangle}{\sqrt{2}}\right], \text { se } f(0)=f(1) \\
\pm|1\rangle \otimes\left[\frac{|0\rangle-|1\rangle}{\sqrt{2}}\right], \text { se } f(0) \neq f(1)
\end{array}\right.
$$

que pode ser convenientemente reescrita como:

$$
\left|\Psi_{3}\right\rangle= \pm[|f(0) \oplus f(1)\rangle] \otimes\left[\frac{|0\rangle-|1\rangle}{\sqrt{2}}\right] .
$$

Com esta maneira de descrever o estado quântico deste registrador após a última aplicação de $H$, fica fácil ver que o estado do primeiro qbit carrega a informação que desejamos a respeito de $f(x)$. Assim se $|f(0) \oplus f(1)\rangle=|0\rangle$ então $f(x)$ é constante, e se $|f(0) \oplus f(1)\rangle=|1\rangle f(x)$ é balanceada. Basta realizar uma única medida sobre o primeiro qbit para encontrarmos a resposta que procurávamos.

Você notou que, na Eq. (7), o que difere as duas possíveis formas de se representar o estado quântico deste registrador, a menos de uma fase global, é apenas um fator de fase relativa introduzido no estado do primeiro qbit? É justamente neste fator de fase que está implícita a resposta para a pergunta central do problema de Deutsch.

Vamos reescrever a Eq. (7) de uma outra maneira para tentar deixar isto mais claro onde novamente, por simplicidade, serão omitidos os fatores de normalização.

$$
\begin{aligned}
\left|\Psi_{2}\right\rangle & =\sum_{x=0,1}(-1)^{f(x)}|x\rangle[|0\rangle-|1\rangle] \\
& =\left[(-1)^{f(0)}|0\rangle+(-1)^{f(1)}|1\rangle\right][|0\rangle-|1\rangle] \\
& =(-1)^{f(0)}\left[|0\rangle+(-1)^{f(0) \oplus f(1)}|1\rangle\right][|0\rangle-|1\rangle] .
\end{aligned}
$$

Nesta nova forma de escrever o resultado anterior é fácil ver que a aplicação de $U_{f}$ ao vetor de estado $\left|\Psi_{1}\right\rangle$ deixa inalterado o estado do segundo qbit mas, introduz um fator de fase relativa igual a $(-1)^{f(0) \oplus f(1)}$ no estado do primeiro qbit. Se $f(x)$ é constante o estado do primeiro qbit será portanto $(|0\rangle+|1\rangle)$, e uma aplicação posterior de $H$ obre este resultará em $|0\rangle$. Por outro lado, se $f(x)$ é balanceada então o estado $(|0\rangle-|1\rangle)$ é levado em $|1\rangle$ pela aplicação de $H$.

Portanto podemos concluir que o acesso à informação desejada sobre $f(x)$, constante ou balanceada, só é possível graças à superposição e à interferência. A interferência é o ingrediente chave do algoritmo de Deutsch que faz com que a resposta procurada seja apresentada de maneira elegante como um fator de fase relativa entre os vetores de estado da base computacional.

\section{Interferômetro de Mach-Zehnder}

Nesta seção abordaremos um dos fenômenos físicos mais notáveis, a interferência. Existem várias aplicações dos princípios de interferometria. Algumas destas são apenas didáticas enquanto outras, são usadas extensivamente em diversas áreas como por exemplo a medicina, odontologia e comunicações ópticas 28.

Típico do movimento ondulatório, este fenômeno pode ser observado em qualquer tipo de onda seja ela mecânica (onda sonora), eletromagnética (onda luminosa) ou onda de matéria (partículas elementares). No caso da luz, existem vários textos que tratam sobre a interferência óptica 29, 30.

O interferômetro de Mach-Zehnder (IMZ) é um bom exemplo de dispositivo gerador de interferência óptica através de divisão de amplitude. Ele foi proposto pela primeira vez em 1891 por Ludwig Zehnder, que estava interessado em estudar o efeito de variações de pressão sobre o índice de refração da água 31]. O mesmo aparato foi novamente proposto um ano depois, de forma independente, por Ludwig Mach como ferramenta para estudar dinâmica de gases não estacionária [32].

Como mostrado no esquema da Figura 3, este aparato é constituído de dois divisores de feixes ( $B S$ do inglês beam 


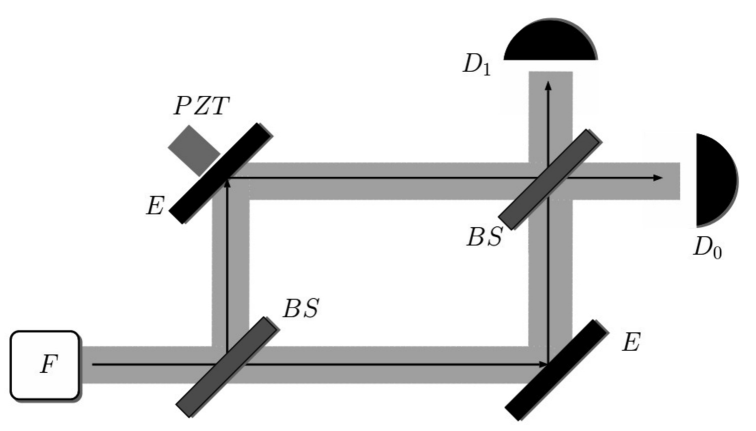

Figura 3: Interferômetro de Mach-Zehnder.

splitter) e dois espelhos totalmente refletores $(E)$. O feixe de luz colimado que emerge da fonte $F$ atinge o primeiro $B S$ e tem sua amplitude igualmente dividida entre outros dois feixes que se propagam em caminhos diferentes de forma que, em termos de conservação de energia, podemos dizer que metade da energia luminosa é transmitida pelo $B S$ e outra metade refletida. Em seguida, os espelhos $E$ redirecionam os dois feixes para a direção do segundo $B S$, onde os feixes irão se superpor e, dependendo da diferença de fase, irão interferir construtiva ou destrutivamente. Desta forma, temos duas detecções possíveis nos detetores $D_{0}$ e $D_{1}$, conforme Figura 3.

Se introduzirmos uma diferença de fase relativa entre os dois feixes que se propagam em caminhos diferentes, como por exemplo alterando o comprimento dos braços deste interferômetro, certamente observaremos um padrão de interferência longitudinal nos detetores e, em geral, um feixe mais intenso em um dos detectores enquanto no outro, um feixe menos intenso. Essa diferença de fase é introduzida pela diferença de caminho óptico, produzida, por exemplo, pelo uso de uma cerâmica piezoelétrica (PZT) em um dos espelho.

As equações para as intensidades dos feixes luminosos que são detectados por $D_{0}$ e $D_{1}$, respectivamente, podem ser facilmente obtidas utilizando o eletromagnetismo clássico 33]:

$$
\begin{aligned}
& I_{0}=I_{T} \cos ^{2}[\Delta \theta] \\
& I_{1}=I_{T} \operatorname{sen}^{2}[\Delta \theta]
\end{aligned}
$$

onde $I_{T}$ é a intensidade total da fonte luminosa, e $\Delta \theta$ é a diferença de fase entre os braços do IMZ. Se o interferômetro é balanceado, ou seja, tem braços com o mesmo comprimento, cabe ao $P Z T$, sob ação de uma variação de tensão controlar, de forma muito fina, a diferença de comprimento entre os braços que percorrerão caminhos diferentes $\vec{r}$ e $\vec{r}$. Essa diferença é da ordem de poucos comprimentos de onda. A defasagem entre os feixes que percorrem os braços do IMZ é dada por

$$
\Delta \theta=\frac{\vec{k} \cdot\left(\vec{r}-\vec{r}^{\prime}\right)}{2},
$$

onde $\vec{k}$ é o vetor de onda.

Em nosso laboratório, realizamos a montagem de um IMZ utilizando um laser de estado sólido com compri- mento de onda de emissão de $532 \mathrm{~nm}$, polarizado horizontalmente. Para introduzir fase relativa entre os dois caminhos, alterando levemente o comprimento de um dos braços do interferômetro, utilizamos um PZT em um dos espelhos $E$, conforme Figura 3. A fonte de tensão utilizada foi controlada por um gerador de sinal que permite que apliquemos uma tensão de forma triangular de baixa frequência sobre o $P Z T$.

Assim, a diferença de fase $\Delta \theta$ varia linearmente em meio período. As intensidades, então, irão oscilar entre os detetores $D_{0}$ e $D_{1}$. Usando fotodetetores, medimos a DDP em cada um deles, que é proporcional a intensidade luminosa detectada. Os resultados são mostrados na Figura 4 Como podemos ver, as intensidades estão em oposição de fase. Ou seja, quando temos um máximo em $D_{0}, D_{1}$ apresenta um mínimo, e vice-versa. Este resultado está em excelente acordo com as Eqs. 13 e nos forneceu dois aspectos muito importantes para simulação experimental do algoritmo de Deutsch: 1) conseguimos controlar a diferença de fase entre os braços de forma muito precisa e 2) conseguimos fazer com que um feixe laser deixe o IMZ praticamente por uma única porta de saída, com visibilidade da ordem de $98 \%$.

\section{Interferômetro de Mach-Zehnder e algoritmo de Deutsch}

Nesta seção vamos apresentar a realização experimental da proposta de Cabral et al [20]. Iniciaremos com uma sumarização desta proposta e passaremos a descrever o experimento. Vale destacar que podemos descrever o circuito como sendo percorrido por um único fóton, porém, o experimento será feito com um feixe intenso, explorando a analogia entre óptica clássica e mecânica quântica [21].

\subsection{O algoritmo de Deutsch e o interferômetro de Mach-Zehnder}

Daqui em diante precisamos reinterpretar, levando em consideração os fenômenos quânticos, os elementos do

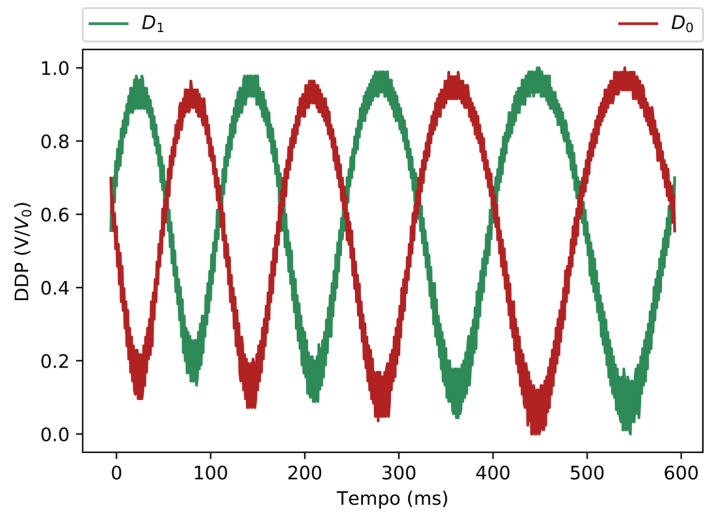

Figura 4: Gráficos das diferenças de potencial (normalizadas) de $D_{0}$ e $D_{1}$ com o uso do gerador de sinais. 
IMZ que são análogos aos elementos dos circuitos de Deutsch mostrados na Seção 3 .

Neste sistema nossos qbits são codificados como os possíveis caminhos a serem percorridos por um fóton, vindo da fonte $F$, que acessa o interferômetro conforme Figura 3. Vale notar que, embora um feixe luminoso possa ser dividido pelo $B S$, um único fóton não pode ser separado desta maneira. Porém, os caminhos disponíveis para o fóton constituem o ingrediente que possibilita o fenômeno da interferência.

Ao representar os possíveis caminhos a serem percorridos por $|0\rangle$ e $|1\rangle$, se um fóton, inicialmente no estado $|0\rangle$, por exemplo, codificado na propagação ao longo direção horizontal mostrada na Figura 3 , incide sobre $B S$, então este tem seu caminho alterado

$$
|0\rangle \rightarrow \frac{1}{\sqrt{2}}(|0\rangle+|1\rangle)
$$

Aqui consideramos que as amplitudes de reflexão e transmissão são iguais $R=T=1 / 2$.

Se, por outro lado, o fóton estivesse no estado $|1\rangle$ (na codificação sugerida, a entrada na direção vertical - porta de entrada vazia do IMZ mostrada na Figura 3):

$$
|1\rangle \rightarrow \frac{1}{\sqrt{2}}(|0\rangle-|1\rangle) \text {. }
$$

Vale notar que, o que distingue os estados 15 e 16 é apenas um fator de fase relativa estre os caminhos $|0\rangle$ e $|1\rangle$. Sendo assim, os $B S$ são responsáveis por criar superposição de estados e introduzir um fator de fase relativa. Esta operação pode ser representada pelo seguinte operador unitário:

$$
U_{B S}=\frac{1}{\sqrt{2}}\left[\begin{array}{cc}
1 & 1 \\
1 & -1
\end{array}\right] .
$$

É fácil ver que 17) satisfaz 15,16 e que $U_{B S}$ atua nos caminhos $|0\rangle$ e $|1\rangle$ de forma idêntica a porta Hadamard atuando em um qbit, que introduzimos anteriormente,

$$
\begin{aligned}
& U_{B S}|0\rangle=\frac{1}{\sqrt{2}}(|0\rangle+|1\rangle) \\
& U_{B S}|1\rangle=\frac{1}{\sqrt{2}}(|0\rangle-|1\rangle)
\end{aligned} .
$$

A ação dos espelhos $E$, representada pelo operador unitário $U_{M}$, é simplesmente a de levar o estado $|0\rangle$ em |1) e vice-versa:

$$
\begin{aligned}
& U_{M}|0\rangle=|1\rangle \\
& U_{M}|1\rangle=|0\rangle
\end{aligned} .
$$

O caminho percorrido por um fóton, partindo do estado $|0\rangle$, pode ser representado por:

$$
\begin{aligned}
|0\rangle & \Rightarrow \frac{1}{\sqrt{2}}(|0\rangle+|1\rangle) \\
& \Rightarrow \frac{1}{\sqrt{2}}\left(e^{i \phi_{0}}|1\rangle+e^{i \phi_{1}}|0\rangle\right) \\
& \Rightarrow \frac{1}{\sqrt{2}}\left(e^{i\left(\phi_{0}-\phi_{1}\right) / 2}|1\rangle+e^{-i\left(\phi_{0}-\phi_{1}\right) / 2}|0\rangle\right) \\
& \Rightarrow \cos \frac{\phi_{0}-\phi_{1}}{2}|0\rangle-i \sin \frac{\phi_{0}-\phi_{1}}{2}|1\rangle \\
& \equiv\left|\Psi_{\text {out }}\right\rangle^{2} .
\end{aligned}
$$

Onde, por simplicidade, foi omitido o fator de fase global $e^{i\left(\phi_{1}+\phi_{0}\right) / 2}$. As probabilidades do fóton inicialmente no estado $|0\rangle$ ser detectado em $D_{0}$ e $D_{1}$ são, respectivamente,

$$
\begin{aligned}
& P_{0}^{0}=\left|\left\langle 0 \mid \Psi_{\text {out }}\right\rangle\right|^{2} \equiv \cos ^{2}\left(\frac{\phi_{0}-\phi_{1}}{2}\right) \\
& P_{1}^{0}=\left|\left\langle 1 \mid \Psi_{\text {out }}\right\rangle\right|^{2} \equiv \sin ^{2}\left(\frac{\phi_{0}-\phi_{1}}{2}\right)
\end{aligned} .
$$

Um desenvolvimento completamente análogo ao anterior, nos leva aos seguintes resultados para o caso em que o fóton se encontra inicialmente no estado $|1\rangle$ :

$$
\begin{aligned}
& P_{0}^{1}=\left|\left\langle 0 \mid \Psi_{\text {out }}\right\rangle\right|^{2} \equiv \sin ^{2}\left(\frac{\phi_{0}-\phi_{1}}{2}\right) \\
& P_{1}^{1}=\left|\left\langle 1 \mid \Psi_{\text {out }}\right\rangle\right|^{2} \equiv \cos ^{2}\left(\frac{\phi_{0}-\phi_{1}}{2}\right)
\end{aligned} .
$$

Aqui é interessante notar que, a menos de uma constante multiplicativa, as expressões de 2122 são iguais as de 13 . Isto mostra que, como já era de se esperar, a probabilidade de detecção está diretamente relacionada com a intensidade luminosa nas saídas do IMZ. Além disso também fica evidente a relação de $\Delta \phi=\phi_{0}-\phi_{1}$ $\operatorname{com} \Delta \theta=\vec{k} \cdot\left(\vec{r}-\vec{r}^{\prime}\right) / 2$.

Para evidenciar o que o IMZ e o circuito de Deutsch possuem em comum, vejamos a execução do algoritmo em paralelo com o caminho percorrido pelo fóton de acordo com as Figuras 2 e 3, assim como feito por Cabral et al 20 .

Criar superposição de estados: no circuito de Deutsch as portas $H$ criam superposição dos estados de entrada, assim como $B S$ coloca o estado de entrada do fóton em uma superposição:

$$
|0\rangle|1\rangle \Rightarrow \frac{1}{2}(|0\rangle+|1\rangle)(|0\rangle-|1\rangle)
$$

Introduzir fase relativa: esta fase é introduzida no algoritmo de Deutsch através da aplicação de $U_{f}$, enquanto que no IMZ esta fase é introduzida defasando-se os caminhos $|0\rangle$ e $|1\rangle$ por $\phi_{0}$ e $\phi_{1}$ :

$$
\Delta \phi \equiv\left(\phi_{0}-\phi_{1}\right)=\left\{\begin{array}{lll}
0 & \text { se } & f(0)=f(1) \\
\pi & \text { se } & f(0) \neq f(1)
\end{array}\right.
$$

Desfazer superposição: a última porta $H$ do circuito de Deutsch remete o estado do primeiro qbit para $|0\rangle$ se $f$ é constante, ou para $|1\rangle$ se $f$ é balanceada. No IMZ o segundo $B S$ recombina os percursos:

$$
|0\rangle+e^{i \Delta \phi}|1\rangle=\cos (\Delta \phi / 2)|0\rangle-i \sin (\Delta \phi / 2)|1\rangle
$$

onde foi omitido o fator de fase global. Assim, se $\Delta \phi=$ $0, \pi$ os estados de saída serão $|0\rangle,|1\rangle$ respectivamente.

Deste modo ficou fácil de ver que cada passo do algoritmo de Deutsch corresponde exatamente a passagem do fóton por um elemento óptico do IMZ e isto está de acordo com a afirmação de Cleve et al 22] de que o IMZ e o algoritmo de Deutsch possuem a mesma estrutura matemática. 


\subsection{Investigação experimental}

Para investigar experimentalmente a interpretação do algoritmo de Deutsch com o IMZ, realizamos a montagem representada na Figura 5 Um feixe laser ESBD ( Estado Sólido Bombeado por Diodo), $532 \mathrm{~nm}, 20 \mathrm{~mW}$ horizontalmente polarizado tem sua intensidade controlada por um filtro neutro $(F N)$ de modo a evitar saturação nas medidas. Trabalhamos com uma intensidade de $1 \mathrm{~mW}$. Um divisor de feixes (BS1) divide o feixe em dois com intensidades balanceadas. A parte transmitida tem sua direção de propagação associada ao qbit $|0\rangle$ incidindo em uma das portas de entrada do IMZ. A parte refletida do feixe é manipulada um jogo de dois espelhos que direciona a luz para a segunda porta do IMZ. Sua propagação em direção ortogonal ao feixe transmitido é associada ao qbit $|1\rangle$. Desta forma podemos estudar as entradas $|0\rangle$ e $|1\rangle$.

A diferença de caminho do IMZ é controlada por uma cerâmica piezoelétrica $(P Z T)$. Este dispositivo será responsável pelo controle da diferença de fase $\Delta \phi$. Em outras palavras, simula $U_{f}$, como discutido na Seção 5.1. O estado representado pelo feixe rotulado no caminho $|0\rangle$ sofrerá as transformações impostas pelo IMZ descritas pelas Eqs. 16, e o feixe do caminho $|1\rangle$, será transformado de forma equivalente, porém, com uma fase de $\pi$ nas componentes refletidas no $B S$. Os dois braços são superpostos no segundo $B S$ do IMZ, que demarca as possíveis saídas do circuito óptico.

As saídas do IMZ são direcionadas para um anteparo onde uma câmera digital $(C C D)$ captura simultaneamente as imagens das duas saídas. Desta forma, podemos associar as imagens de cada saída aos detetores $D_{0}$ e $D_{1}$.

O IMZ é alinhado para a entrada $|0\rangle$. A tensão no $P Z T$ é reduzida ao mínimo e aumenta-se até que o primeiro máximo em $D_{0}$ seja observado. Esta tensão define $\Delta \phi=0$, ou seja, a entrada $|0\rangle$ permanece no caminho $|0\rangle$. Aumentando a tensão no $P Z T$ pode-se atingir uma defasagem $\Delta \phi=\pi$, fazendo com que a entrada $|0\rangle$ troque

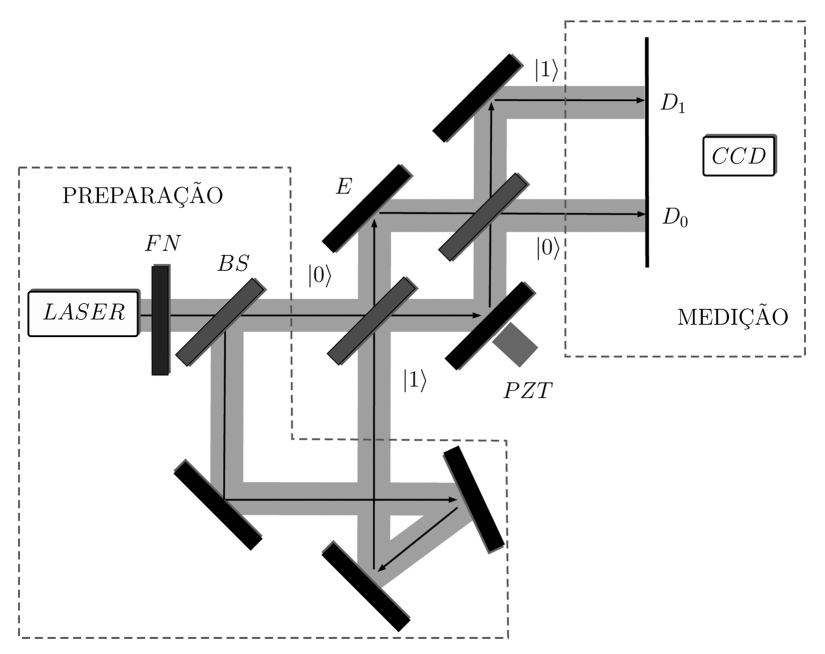

Figura 5: Esquema experimental. a porta de saída, iluminando a área identificada com o detector $D_{1}$.

Vamos analisar o experimento apenas com a entrada $|0\rangle$. Neste caso, bloqueamos a entrada $|1\rangle$. Ajustando a tensão no $P Z T$ correspondente à $\Delta \phi=0$, vemos que só a região associada a $D_{0}$ é iluminada como pode ser visto na primeira linha da imagem da Figura 6 referente à entrada zero. Este resultado está em excelente acordo com a probabilidade $P_{0}^{0}$ dada pela Eq.21. O ajuste do $P Z T$ é feito manualmente por uma fonte de alta tensão com ajuste fino e fica estável por cerca de 30 segundos, tempo suficiente para registro da imagem. Isso simula o resultado para o caso de função constante, como discutido na seção 5.1. Note que neste caso fazemos o análogo à medida do qbit de saída. Vale salientar que para termos intensidade nula na saída associada à $D_{1}$, atenuamos o feixe de modo que o mínimo de interferência ficasse abaixo da sensibilidade da câmera. A visibilidade do IMZ foi medida em $98 \%$.

Para verificar o caso em que a entrada é $|1\rangle$, bloqueamos a entrada $|0\rangle$ e, com o conjunto de espelhos, alinhamos o feixe referente à entrada $|1\rangle$ no IMZ. Para a tensão associada à $\Delta \phi=0$, este feixe é observado na saída associada a $D_{1}$, também em excelente acordo como a probabilidade $P_{1}^{1}$ dada pela Eq 22

Controlando o PZT, podemos introduzir uma diferença de fase $\Delta \phi=\pi$, condição para simulação do resultado para função balanceada. A Figura 7 mostra o resultado experimental. Para entrada $|0\rangle$, somente a saída $D_{1}$ apresenta intensidade relevante, mostrando que o qbit de entrada $|0\rangle$ foi convertido em $|1\rangle$. Assim, os resultados experimentais corroboram a simulação proposta pela Ref. 20. Para entrada $|1\rangle$, vemos que o qbit também inverte, sendo observado na área referente ao detetor $D_{0}$, o que evidencia a independência do estado de entrada. O protocolo mantém o qbit de entrada para o caso constante e inverte-o para o caso de função balanceada. Estes resultados estão de acordo com as probabilidades $P_{1}^{0} \mathrm{e}$ $P_{0}^{1}$, dadas, respectivamente, pelas Eqs. 21 e 22

Vamos discutir agora uma limitação desta interpretação. Na simulação proposta por Cabral et al. [20], a

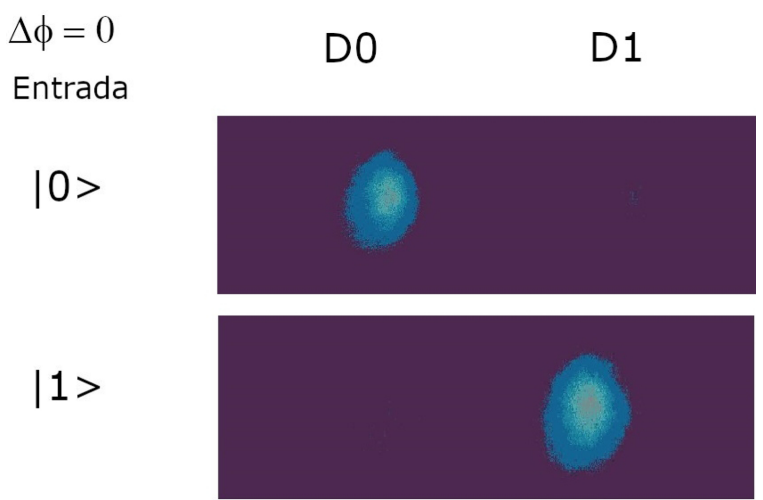

Figura 6: Resultado para entradas $|0\rangle$ e $|1\rangle$ para o caso em que $\Delta \phi=0$. As intensidades estão representadas em falsecolor. 


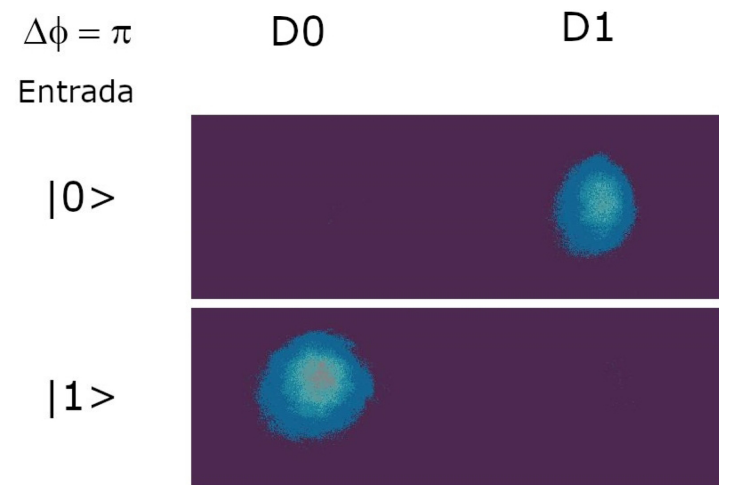

Figura 7: Resultado para as entradas $|0\rangle$ e $|1\rangle$ para o caso em que $\Delta \phi=\pi$. As intensidades estão representadas em falsecolor.

interpretação do algoritmo de Deutsch pelo IMZ se dá pelo fato de que, na prática, $U_{f}$ introduz uma fase relativa entre os caminhos 0 e 1 do qbit $|0\rangle$. No algoritmo de Deutsch essa diferença de fase depende do cálculo da função $f$, cujo resultado depende do segundo qbit $\left(U_{f}|x\rangle|y\rangle=|x\rangle|y \oplus f(x)\rangle\right)$. É pelo segundo qbit estar em uma superposição produzida pela porta Hadamard no qbit $|1\rangle$ que aparece o termo $(-1)^{f(0) \oplus f(1)}$, conforme Eq.4 Porém, nenhum cálculo de $U_{f}$ é feito no IMZ. Na simulação proposta na Ref. [20], a diferença de fase é adicionada pelo controle da distância de propagação nos braços de IMZ com o PZT, independente do segundo qbit. Por este motivo, podemos analisar somente o primeiro qbit, ou seja, medir o primeiro qbit, conforme também é feito no algoritmo de Deutsch.

\section{Conclusões}

Neste trabalho mostramos a realização experimental da simulação do algoritmo de Deutsch com o interferômetro de Mach-Zehnder, proposta na Ref. [20]. Os resultados experimentais estão em excelente acordo com as previsões teóricas. O aparato proposto permite a simulação com a entrada de dois qbits diferentes. O resultado desta simulação mostrou também a limitação da interpretação do algoritmo de Deutsch através do IMZ. Os resultados são bem emulados pelo circuito óptico, porém, diferentemente do circuito de Deutsch, independem do segundo qbit. Este resultado implica dizer que o algoritmo de Deutsch foi simulado e não implementado pelo IMZ. Esta conclusão é a mesma se o experimento fosse realizado com fótons únicos, uma vez que na analogia de feixes intensos as intensidades medidas normalizadas estão associadas às probabilidades de detecção em cada porta. Desta forma, portas não iluminadas no experimento com feixes intensos equivalem a probabilidades nulas de detecção de fótons únicos. Essa analogia tem sido muito utilizada na literatura para estudar princípios de informação quântica sendo um cenário muito promissor para discussão e ensino de aspectos fundamentais da mecânica quântica.

\section{Referências}

[1] M.A. Nielsen e I.L. Chuang, Quantum Computation and Quantum information (Cambridge University Press, Cambridge, 2000).

[2] D. Deutsch, Proc. R. Soc. A 400, 97 (1985).

[3] P.W. Shor, Proc. A. Symp. Found. Comp. Sci. 35, 124 (1994).

[4] L.K. Grover, Proc. A. ACM. Symp. Theor. Comp. 28, 212 (1996).

[5] R.P. Feynman, Int. J. Theor. Phys. 21, 6 (1982).

[6] S. Jordan, Quantum algorithm zoo, disponível em http://math.nist.gov/quantum/zoo/, acessado em 26/10/2018.

[7] R. Blatt e C.F. Roos, Nat. Phys. 8, 277 (2012).

[8] I.S. Oliveira, T.J. Bonagamba, R.S. Sarthour, J.C. Freitas e E.R. Azevedo, NMR Quantum Information Processing (Elsevier, Oxford, 2007).

[9] H. Bachor e T.C. Ralph, A Guide to Experiments in Quantum Optics (Wiley-VCH, Weinheim, 2004).

[10] D. Gloge e D. Marcuse, J. Opt. Soc. Am. 59, 1629 (1969).

[11] S. Goyal, T. Konrad, em: Laser Beam Propagation: Generation and Propagation of Customized Light editado por A. Forbes (CRC Press, Florida, 2014).

[12] D. Stoler, J. Opt. Soc. Am. 71, 334 (1981).

[13] C.V.S. Borges, M. Hor-Meyll, J.A.O. Huguenin e A.Z. Khoury, Phys. Rev. A 82, 033833 (2010).

[14] K.H. Kagalwala, G. Di Giuseppe, A.F. Abouraddy e B.E.A. Saleh, Nat. Photon. 7, 72 (2012).

[15] W.F. Balthazar, C.E.R. Souza, D.P. Caetano, E.F. Galvão, J.A.O. Huguenin e A.Z. Khoury, Opt. Lett. 41, 5797 (2016).

[16] W.F. Balthazar, D.P. Caetano, C.E.R. Souza e J.A.O. Huguenin, Rev. Bras. Ens. Fís. 44, 658 (2014).

[17] A.C. Santos, Rev. Bras. Ens. Fís. 39, 1, e1301 (2017).

[18] M.A. José, J.R. Piqueira e R.D. Lopes, Rev. Bras. Ens. Fís. 35, 1306 (2013).

[19] W.R.M. Rabelo e M.L.M. Costa, Rev. Bras. Ens. Fís. 40, e4306 (2018).

[20] G.M. Cabral, A.F. de Lima e B. Lula Jr., Rev. Bras. Ens. Fís. 26, 109 (2004).

[21] A.L.P. Camargo, L.O. Pereira, W.F. Balthazar e J.A.O. Huguenin, Rev. Bras. Ens. Fís 39, e2305 (2017).

[22] R. Cleve, A. Ekert, C. Macchiavello e M. Mosca, Proc. R. Soc. Lond. A 454, 339 (1998).

[23] L. Diosi, A Short Course in Quantum Information Theory: An Approach from Theoretical Physics (SpringerVerlag Berlin Heidelberg, Heidelberg, 2011).

[24] J. Stolze e D. Suter, Quantum Computing: A Short Course from Theory to Experiment (Wiley-VCH, Weinheim, 2008).

[25] D. Griffiths, Quantum Mechanics (Pearson, Londres, 2011).

[26] J. Kinne, Deutsch's algorithm, disponível em http:// www.cs.xu.edu/ kinne/quantum/deutche.html, acessado em 29/01/2018.

[27] D. Deutsch, Proc. R. Soc. A 425, 73 (1989).

[28] M.T. Carvalho, Técnicas de Interferometria óptica aplicada à medicina, odontologia e comunicações ópticas. Tese de Doutorado, Universidade Federal de Pernambuco (2006). 
[29] H.M. Nussenzveig, Curso de Física Básica (Edgarg Blücher, São Paulo, 1998).

[30] E. Hecht, Optics (Addison-Wesley, San Francisco, 2002).

[31] L. Zehnder, Z. Instrum. kd. 11, 275 (1891).

[32] L. Mach, Z. Instrum. kd. 12, 89 (1982).

[33] J.D. Jackson, Classical Electrodynamics (Wiley, New York, 1998), 3rd Ed. 